\title{
LA DIRECCIÓN DEL PROCESO DE ENSEÑANZA APRENDIZAJE DE LAS MATEMÁTICAS: MATERIAL DIDÁCTICO
}

\author{
The Direction of the Teaching Learning Process of Mathematics: Didactic Material
}

\author{
Adailsis Jerez Aguilar, MsC. \\ Universidad de Oriente, Cuba \\ https://orcid.org/0000-0003-2868-7564 \\ ada.jerez@uo.edu.cu
}

\author{
Milagros Gutiérrez Sánchez, MsC. \\ Universidad de Oriente, Cuba \\ https://orcid.org/0000-0001-7146-250X \\ milagros.gutierrez@uo.edu.cu
}

\author{
Maribel Rodríguez Núñez, Dra. C. \\ Universidad de Oriente, Cuba \\ https://orcid.org/0000-0003-4921-7115 \\ maribelrn@uo.edu.cu
}

Palabras claves: Enseñanza - Aprendizaje, Material Didáctico.

Keywords: Teaching - Learning, Teaching Materials.
Recibido: 26 de noviembre de 2019

Aceptado: 18 de diciembre de 2019

\section{RESUMEN}

Las exigencias actuales para el desarrollo exitoso del proceso de enseñanza aprendizaje en la escuela primaria requieren de un desempeño metodológico por tal razón, se propone un material didáctico donde se encuentran propuestas de ejercicios contenidos en los textos Para ti maestro, los "cuadernillos" empleados en los operativos de la medición de la calidad, así como otros creados con fines didácticos. El material incluye, además, los objetivos que deben ser vencidos en cada período, así como los mínimos esenciales de las unidades del programa. También se ofrecen orientaciones metodológicas específicas para el trabajo del profesor universitario, de los docentes en formación, y de los maestros en funciones; sobre contenidos que no están explicados en los documentos normativos existentes. Esta propuesta de actividades contribuye a sistematizar y evaluar los diferentes dominios cognitivos.

\section{ABSTRACT}

The current requirements for the successful development of the teaching-learning process in the primary school require a methodological performance for such reason, a didactic material is proposed where there are proposals of exercises contained in the texts For you master, the "booklets" used in the operatives of the measurement of the quality, as well as others created with didactic aims. The material also includes the objectives that must be overcome in each period, as well as the essential minimums of the program units. Specific methodological guidelines are also offered for the work of the university professor, of the teachers in training, and of the teachers in functions; about contents that are not explained in the existing normative documents. This proposal of activities contributes to systematize and evaluate the different cognitive domains. 


\section{INTRODUCCIÓN}

Como resultado del acelerado desarrollo científico técnico, la educación cubana actual tiene ante sí varios retos dirigidos a la formación integral de los escolares desde edades tempranas. Uno de estos es concebir el proceso de enseñanza aprendizaje de manera que el alumno asuma una posición activa en la escuela, que esta le motive, le estimule a pensar, a suponer y valorar, le plantee un trabajo intelectual a su alcance y progresivamente exigente.

El perfeccionamiento educacional que tiene lugar en Cuba y muy especialmente en la Educación Primaria reclama de una escuela que se caracterice por su aporte al desarrollo de la cultura general integral de los alumnos; que significa garantizar en cada uno de ellos el saber, saber hacer y saber ser, a partir de su formación como individuos activos, reflexivos, creativos, independientes y libres.

Por tanto se requiere de un maestro con un alto nivel de preparación y maestría pedagógica que le permita realizar un trabajo sistémico en todas las asignaturas del currículo de estudio fundamentalmente en Matemática, que es una de las que permite estimular la actividad intelectual del educando, desarrollar la capacidad de análisis reflexivo y creativo, preparar al individuo para la vida permitiéndole hacer valoraciones con independencia, o sea un maestro que sea capaz de elaborar un sistema de clases con calidad.

En el análisis desarrollado, a partir de la experiencia acumulada en la dirección del trabajo metodológico de la Educación Primaria, se ha profundizado en el tema evidenciándose que aún cuando se constatan avances, existen insuficiencias para desarrollar un proceso eficiente de enseñanza-aprendizaje en la asignatura Matemática, cualitativamente superior.

Una de las direcciones fundamentales del trabajo metodológico del Ministerio de Educación, plantea la preparación del personal docente y en formación con la calidad requerida como pilares fundamentales que sustentan la preparación del colectivo docente en cada nivel de enseñanza, para lograrlo se adoptan medidas organizativas encaminadas a garantizar que el alumno reciba la atención que requiere, tanto para eliminar las insuficiencias en su preparación precedente como las que surjan en el aprendizaje de los nuevos contenidos.

En la literatura científica se advierte la importancia que tiene la Didáctica para el desempeño profesional en la educación. Al respecto, se analiza el conocimiento didáctico del contenido pues este permite al docente transferir a sus estudiantes el conocimiento que tienen sobre un contenido disciplinar.

Por otra parte, se precisa que revela la aplicación del conocimiento didáctico y pedagógico de carácter general a la enseñanza de una disciplina en particular; su desarrollo ocurre a través de la experiencia y la práctica en el aula y debería ser eje central de la formación profesional.

De igual forma, se plantea que el conocimiento didáctico del contenido se expresa en las relaciones entre el conocimiento que posee el profesor de una disciplina específica con la manera de enseñarla. En las ideas anteriores se precisa que la formación didáctica implica: el dominio del contenido de cada ciencia, el dominio de la didáctica general, la integración de los contenidos de la ciencia a enseñar y de los didácticos, así como del sistema de experiencias profesionales.

De ahí que las autoras de este trabajo ofrecen un material didáctico para el trabajo del profesor universitario, de los docentes en formación, y de los maestros en funciones; con vista al mejor desarrollo del proceso de enseñanzaaprendizaje de que se trate.

Este material resume aspectos de las Orientaciones Metodológicas, del Programa, de la programación televisiva, ajustes curriculares, entre otros; de modo que viabilice la planificación del docente.

En este material aparecen los documentos normativos que se deben consultar para la auto preparación y preparación de los sistemas de clases, que le posibilita el perfeccionamiento del proceso, por tanto este se constituye en vía metodológica para todos los implicados.

El mismo puede ser utilizado por la escuela en las preparaciones metodológicas, talleres, preparaciones de asignaturas, encuentros presenciales, entre otras actividades.

La pertinencia del trabajo está dada en la necesidad de elevar la calidad del proceso de enseñanza aprendizaje en correspondencia con las nuevas y crecientes exigencias de la sociedad, si el fin de la educación primaria es formar integralmente a las nuevas generaciones y prepararlas con un alto nivel de desarrollo cognitivo. 


\section{DESARROLLO}

Las matemáticas son un producto del quehacer humano y su proceso de construcción está sustentado en abstracciones sucesivas. Muchos aportes importantes de esta disciplina han partido de la necesidad de resolver problemas concretos, propios de los grupos sociales. Por ejemplo, los números, tan familiares para todos, surgieron de la necesidad de contar y son también una abstracción de la realidad que se fue desarrollando durante largo tiempo. Este desarrollo está; además, estrechamente ligado a las particularidades culturales de los pueblos: todas las culturas tienen un sistema para contar, aunque no todas cuenten de la misma manera.

Los alumnos en la escuela primaria deben adquirir conocimientos básicos de las matemáticas y desarrollar capacidades y habilidades que les posibiliten la elevación de la calidad del aprendizaje, por ello es indispensable que los alumnos se interesen y encuentren significado y funcionalidad en el conocimiento matemático, que lo valoren y hagan de él un instrumento que les ayude a reconocer, plantear y resolver problemas en diversos contextos de su interés.

En los programas de Matemática de la escuela primaria cubana se definen los objetivos generales y específicos que se deben lograr con los estudiantes, así como las habilidades matemáticas a desarrollar. Ello constituye el punto de partida del trabajo del maestro encaminado a lograr el mayor desarrollo posible de sus alumnos, tanto el nivel de conocimientos, como de habilidades, capacidades y valores. (1)

El éxito en el aprendizaje de esta disciplina depende, en buena medida, del diseño de actividades que promuevan la construcción de conceptos a partir de experiencias concretas en la interacción con los otros. En esas actividades, las matemáticas constituyen herramientas funcionales y flexibles que permiten a los escolares resolver las situaciones que se les planteen. Todo ello se ha tenido en cuenta para la elaboración del material didáctico que a continuación se presenta:

\section{Estimado docente:}

Se le propone un material didáctico que puede resultar útil, orientador y con carácter integrador para la preparación de la asignatura Matemática 6. grado, en lo que a objetivos, contenidos y actividades se refiere, para su mejor tratamiento tanto en los docentes en formación como en los maestros de experiencia.

Este material contiene los objetivos que deben ser vencidos en cada período así como los mínimos esenciales de las unidades del programa. Se proponen algunos ejercicios contenidos en el texto Para ti maestro, en los "cuadernillos" empleados en los operativos de la calidad aplicados, así como otros creados con fines didácticos y que presentan interés particular en relación con el contenido que se trata.

Es importante aclarar que este material didáctico, no pretende de ningún modo sustituir el empleo del Programa, Orientaciones Metodológicas, libros de texto, ni ninguno de los documentos normativos vigentes y establecidos por el Ministerio de Educación, sino es una vía metodológica para facilitar la preparación del docente en el desempeño de su labor, todo lo cual asegura eficiencia en la dirección del proceso de enseñanza aprendizaje.

\section{Objetivos que deben lograr los alumnos de 6. Grado por períodos}

Al concluir el I período los alumnos de 6. Grado deben:

1. Desarrollar habilidades en la descomposición de números naturales en factores primos, y las apliquen en la determinación del mínimo común múltiplo de dos o más números naturales, cuando no es posible determinarlos por simple inspección.

2. Resolver las cuatro operaciones fundamentales y lo apliquen a la solución de ejercicios donde se combinen las cuatro operaciones incluyendo la potenciación, la radicación así como ejercicios con texto y problemas.

3. Reafirmar y profundizar el concepto de fracción y su significado práctico, continuar desarrollando habilidades en la comparación y en la realización de operaciones de adición y sustracción con fracciones comunes así como iniciar el desarrollo de habilidades de cálculo en la multiplicación y división de fracciones comunes aplicada a la solución de problemas típicos de fracciones

4. Sistematizar algunas propiedades básicas (axiomas) de la Geometría plana.

Al concluir el II Período los alumnos de 6. grado deben 
1. Reafirmar y profundizar el concepto de fracción y su significado práctico, continuar desarrollando habilidades en la comparación y en la realización de operaciones de adición y sustracción con expresiones decimales así como iniciar el desarrollo de habilidades de cálculo en la multiplicación y división de expresiones decimales aplicada a la solución de problemas y ejercicios con texto

2. Resolver ecuaciones lineales con una variable y lo apliquen a la solución de ejercicios con textos y problemas sencillos.

3. Profundizar en los conocimientos adquiridos en quinto grado sobre los movimientos y en especial conocer que la realización sucesiva (composición) de dos o más movimientos es también un movimiento.

4. Dominar las propiedades especiales de las rectas en los movimientos estudiados y utilizarlas en ejercicios de reconocimiento y argumentación.

5. Dominar las relaciones entre ángulos aplicándolo en ejercicios de reconocimiento, cálculo y argumentación.

6. Reconocer en ilustraciones dadas pares de ángulos correspondientes, alternos y conjugados a partir de su caracterización posicional aplicándolo en ejercicios de reconocimiento, cálculo y argumentación.

Al concluir el III Período los alumnos de 6. Grado deben

1. Calcular un término de una proporción conocidos los tres restantes, reconozcan magnitudes directa e inversamente proporcionales y resuelvan ejercicios de aplicación así como problemas de proporcionalidad.

2. Traducir del lenguaje común al algebraico y viceversa y utilizar estas habilidades en la interpretación y modelación de ejercicios con texto y problemas.

3. Resolver ejercicios formales, con texto y problemas, utilizando una vía algebraica.

Al concluir el IV Periodo los alumnos de 6. Grado deben

1. Interiorizar el concepto de tanto por ciento, identificándolo con partes que se toman de cada 100 y que sean capaces de aplicarlo en problemas de la práctica y puedan reconocer la importancia de la Matemática para reflejar aspectos cuantitativos de la realidad objetiva.

2. Dominar las relaciones entre los ángulos que se forman cuando dos rectas son cortadas por una secante y utilizarla en ejercicios de reconocimiento, cálculo y argumentación.

3. Reconocer los triángulos según sus características (Atendiendo a la longitud de sus lados y a la amplitud de su ángulos) y dominar las relaciones entre lados y ángulos de un triángulo. En particular deben dominar la propiedad de la desigualdad triangular y aplicarla a ejercicios geométricos.

4. Dominar las relaciones que existen entre los ángulos de un triángulo y emplearlo en ejercicios de reconocimiento, cálculo y argumentación.

5. Obtener la fórmula del volumen de un ortoedro, mediante conteo, utilizando un cubo unidad $\left(1 \mathrm{~cm}^{2}\right)$ y emplear el metro cúbico y sus submúltiplos en conversiones y en el cálculo del volumen de ortoedros.

6. Sistematizar las unidades de capacidad y reconocer las relaciones que existen entre ellas y las de volumen.

7. Aplicar los conocimientos adquiridos sobre las unidades de volumen y capacidad en la solución de ejercicios con texto y problemas, teniendo en cuenta las reglas del cálculo aproximado donde se requieran.

8. Utilizar convenientemente el lenguaje y la simbología conjuntista relacionada con la geometría.

\section{Orientaciones metodológicas}

Habilidades más afectadas con respecto al dominio cognitivo geometría 
1. Reconocimiento de las propiedades de las figuras y cuerpos geométricos a fin de aplicarlos en ejercicios propuestos.

2. Realizar demostraciones sencillas a partir de la argumentación y el establecimiento de relaciones geométricas.

3. Identificar cantidad de figuras contenidas en otras.

4. Calcular área y perímetro de figuras en el plano

Propuestas de ejercicios que se relacionan con estas habilidades

1- Ernesto quiere formar triángulos separados con varillas que tienen diferentes longitudes, agrupándolas del siguiente modo.
a) $6 \mathrm{~cm} . ; 8 \mathrm{~cm} . ; 10 \mathrm{~cm}$.
b) $11 \mathrm{~cm} . ; 17 \mathrm{~cm} . ; 9 \mathrm{~cm}$.
c) $15 \mathrm{~cm} . ; 7 \mathrm{~cm} . ; 5 \mathrm{~cm}$.
d) $10 \mathrm{~cm} . ; 9 \mathrm{~cm} . ; 6 \mathrm{~cm}$.

Señale el trío de varillas que no le sirven para formar un triángulo. Fundamente.

$\mathrm{R} /$ No es posible formar un triángulo con las dimensiones del inciso $\mathrm{c}$ )

Porque no se cumple el teorema de la desigualdad triangular que plantean que la longitud de cada lado debe ser mayor que la suma de los otros dos lados. Ejemplo: $15>7+5$

2- Sean $A$ y $B$ dos ángulos adyacentes, tal que $A=5 x+85^{\circ}$ y $B=4 x+68^{\circ}$. Determina la amplitud de los ángulos $A$ y $\mathrm{B}$

$$
\begin{array}{rrrrr}
\text { Solución: } & A+B=180^{\circ} & \text { (sustituyendo por sus valores) } \\
5 x+85^{\circ}+4 x+68^{\circ} & =180^{\circ} & & \\
5 x+4 x & =180^{\circ}-85^{\circ}-68^{\circ} & A=5 x+85^{\circ} & B=4 x+68^{\circ} \\
9 x & =27^{\circ} & A=5(3)+85^{\circ} & B=4(3)+68^{\circ} \\
X & =27^{\circ}: 9 & A=100^{\circ} & B=80^{\circ} \\
X=3 & R /: & A=100^{\circ} & B=80^{\circ}
\end{array}
$$

2. Orientaciones metodológicas

Elementos para la definición constructiva de los movimientos

La traslación

Para realzar un movimiento de traslación en el plano se determina:

a) La figura objeto de traslación

b) El vector de traslación

Descripción de la construcción de la traslación en el plano.

a) Se trazan rayos que tengan como origen los puntos denotados en la figura y que sean paralelas al vector de traslación

b) Se transporta la longitud del vector sobre los rayos, con igual sentido.

c) Se denotan los puntos imágenes, se unen y se obtiene la figura imagen. 
Propiedades distintivas del movimiento de traslación en el plano.

Los segmentos determinados por un punto y su imagen:
a) Tienen igual longitud que el vector de traslación
b) Tienen igual dirección (es decir es paralelo) al vector de traslación.
c) Tienen igual sentido que el vector de traslación.

\section{La reflexión}

Para realizar una reflexión en el plano se necesita determinar:

a) La figura objeto de reflexión.

b) El eje de reflexión.

Descripción de la construcción de una reflexión en el plano.

a) Se tazan rayos que tengan como origen los puntos denotados en la figura y que corten al eje perpendicularmente.

b) Se transporta la distancia de cada punto origen al eje sobre el rayo a partir de dicho eje.

c) Denotamos los puntos imágenes, se unen y se obtiene la figura imagen.

Propiedades distintivas del movimiento de reflexión en el plano:

a) Los segmentos determinados por un punto y su imagen, cortan al eje perpendicularmente.

b) La distancia de cada punto y su imagen al eje, es la misma.

c) Los puntos situados en el eje, coinciden con su imagen.

\section{La simetría central}

Para realizar un movimiento de simetría central en el plano, es necesario determinar:

a) La figura objeto de simetría central.

b) El centro de simetría.

Descripción de la construcción de la simetría central.

a) Se trazan rayos que tengan como origen los puntos denotados en la figura y que pasen por el centro de simetría.

b) Se transporta la distancia de cada punto origen al centro de simetría sobre cada rayo a partir del centro de simetría.

c) Denoto los puntos imágenes, se unen y obtenemos la figura imagen.

Propiedades distintivas del movimiento en el plano simetría central.

a) La distancia de cada punto y su imagen al centro de simetría es la misma.

b) Los segmentos determinados por un punto y su imagen se cortan en su punto medio que coincide con el centro de simetría.

c) Cada punto y su imagen tienen un ángulo de rotación de 180 grados por lo que cada punto y su imagen se encuentran contenidos en una misma recta. 


\section{La rotación}

Para realizar un movimiento de rotación en el plano se necesita determinar
a) La figura objeto de rotación
b) El centro de rotación
c) El ángulo de rotación
d) El sentido de la rotación.

Descripción de la construcción de un movimiento de rotación en el plano.

a) Se trazan rayos que tengan como origen el centro de rotación y que pasen por los puntos denotados en la figura.

b) Se traza en el ángulo un arco de circunferencia.

c) Con igual amplitud, se transporta el arco de circunferencia haciendo (con el compás) centro en el centro de rotación.

d) Se toma con el compás la longitud del arco.

e) Haciendo centro con el compás, donde se cortan el arco de circunferencia con los rayos, se transporta sobre el arco, la longitud del arco del ángulo de rotación.

f) Se trazan los rayos imágenes, con origen en el centro de rotación y que pasen por la intercepción de los arcos.

g) Haciendo centro en el centro de rotación, transporto los puntos orígenes a los rayos imágenes.

h) Se denotan los puntos imágenes, se unen y obtenemos la figura imagen.

Propiedades distintivas del movimiento de rotación en el plano.

a) Todos los puntos de la figura rotan igual ángulo.

b) La distancia de cada punto y su imagen al centro de rotación es la misma.

c) Los puntos situados en el centro de rotación coinciden con su imagen.

\section{Niveles de dificultades}

Para la traslación.

- Que la flecha de traslación esté fuera de la figura, con diferentes direcciones para el vector de dirección.

- Que el vector de reflexión parta de un punto de la figura, teniendo en cuenta diferentes sentidos.

- La composición de este movimiento.

Reflexión.

- Que el eje esté en diferentes posiciones.

- La composición de este movimiento.

Rotación.

- Que el centro de rotación esté en un vértice de la figura, dentro de la figura

- La composición de este movimiento 


\section{Orientaciones metodológicas.}

Para el tratamiento a las magnitudes hay que tener en cuenta las habilidades a lograr (estimar, medir, convertir) y para un adecuado tratamiento de las mismas se proponen sucesiones de indicaciones.

Sucesiones de indicaciones para estimar:

- Observa el objeto a estimar.

- Selecciona la unidad con la que vas a efectuar la estimación.

- Compara mentalmente las veces que está contenida esa unidad en el objeto.

- Escribe el resultado de la estimación.

- Mide utilizando el instrumento adecuado y la misma unidad utilizada en la estimación

- Escribe el resultado de la medición.

- Compara ambos resultados.

- Valóralos.

Sucesión de indicaciones para medir:

- Observa lo que vas a medir.

- Piensa el las unidades que conoces.

- Selecciona la más adecuada.

- Escoge le instrumento de medición.

- Mide.

- Expresa el dato de magnitud.

Sucesiones de indicaciones para convertir:

- Observa la unidad en que te han dado el dato de magnitud.

- Observa la unidad en que debes expresar el dato.

- Compáralas y piensa en le número de conversión.

- Si la unidad que te dan es menor que la que te piden, divide.

- Si la unidad que te dan es mayor que la que te piden, multiplica.

- Realiza la operación.

- Coordina el número de medida con la unidad requerida.

1. Orientaciones metodológicas.

En la unidad temática \# 4 Proporcionalidad correspondiente al tercer período. 
En el epígrafe relacionado a "Magnitudes directamente proporcionales. Relaciones y aplicaciones en ejercicios y problemas el las Orientaciones Metodológicas del grado página 92, se analiza el ejercicio 17 del L/T 6. Grado página 143.

Si una llave vierte 354 litros en un cuarto de hora. ¿Cuántos litros vierte en 5 min?

Litros de agua

354

$\mathrm{X}$

Tiempo en (min.)

15

5

Este ejercicio solo se explica de la siguiente forma:

a) $354 / x=15 / 5 ; 15 x=354.5 ; \quad x=354: 3$

$x=118$

El factor de proporcionalidad es $15 / 3=3$

Se puede resolver de otras maneras aplicando las propiedades de los términos de una proporción, ejemplo:

b) $354 / 15=x / 5 ; 15 x=354.5 ; \quad x=354.5 / 15$

$$
X=118
$$

El factor de proporcionalidad es $354 / 15=23,6$

c) $x / 354=5 / 15 ; 15 x=5.354 \quad x=5.354 / 15$

$$
x=118
$$

El factor de proporcionalidad es $5 / 15=1 / 3$

d) $15 / 354=5 / x ; 15 x=354.5 \quad x=354.5 / 15$

$$
x=118
$$

El factor de proporcionalidad es $15 / 354 \approx 0,04$

2. Orientaciones metodológicas.

Al darle tratamiento al ajuste curricular tratamiento a la información o dominio estadístico, para un mejor tratamiento se debe orientar a los estudiantes de forma clara y precisa para que pueda interpretar los gráficos con destreza, razón por la cual se ofrecen algunas sugerencias de indicaciones y propuestas de ejercicios.

Interpretación de gráficos:

Para favorecer el análisis e interpretación de la información que se sintetiza en el gráfico, el maestro ofrecerá impulsos didácticos para facilitar la comprensión. Puede seguir los pasos que se recomiendan a continuación:

$\checkmark \quad$ Leer el texto para determinar exactamente qué está siendo representado.

$\checkmark \quad$ Analizar la información que se representa.

$\checkmark$ Reconocer las tablas o tipo de gráfico y determinar qué significan.

$\checkmark$ Interpretar los datos. 


\section{$\checkmark$ Arribar a conclusiones.}

$\checkmark$ Teniendo en cuenta las características de la información, elaborar otras formas de representación.

Teniendo en cuenta las características de cada gráfico y las condiciones previas, se recomienda presentar este contenido en el siguiente orden: gráfico de barra, gráfico de línea y gráfico circular.

En la fase de control, el maestro prestará especial atención a:

- La interpretación adecuada de la información cuantitativa a partir de los conocimientos matemáticos que el alumno posee.

- La expresión clara y coherente de las ideas.

- Las valoraciones que se pueden realizar en correspondencia con la situación que se describe y que contribuyen a la formación integral de la personalidad de los alumnos.

Propuesta de ejercicios

2- En la figura se muestran los equipos que participarán en una competencia y la cantidad de integrantes.

\section{Cantidad}

de

integrantes

Equipos

$\begin{array}{lllll}1 & 2 & 3 & 4 & 5\end{array}$

Cuál de las respuestas es la correcta respecto al promedio y la modalidad.

- El promedio de participantes por equipos es 19 y la modalidad es 15.

- El promedio de participantes es 16 y la modalidad es 18.

- La modalidad es 15 y el promedio de participantes 16.

- La modalidad es 17 y el promedio 16.

8- María tenía $5 / 8 \mathrm{lb}$. de chocolate y gasto $2 / 5 \mathrm{lb}$., como manifiesta la parte sombreada. Observa el gráfico y marca la afirmación que consideres correcta respecto a la parte que María gastó de la libra de chocolate.

$2 / 5 \mathrm{lb}$.

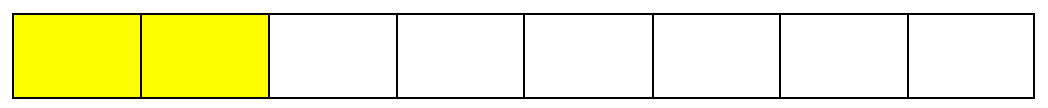

$1 \mathrm{lb}$.

$5 / 8 \mathrm{lb}$.

María gastó:

$>$ La segunda parte de la libra

$>$ La tercera parte de la libra

$>$ La cuarta parte de la libra 
$>$ La octava parte de la libra

9-. El gráfico representa los resultados de una encuesta realizada a 35 alumnos de sexto grado sobre su preferencia por algunos programas infantiles.

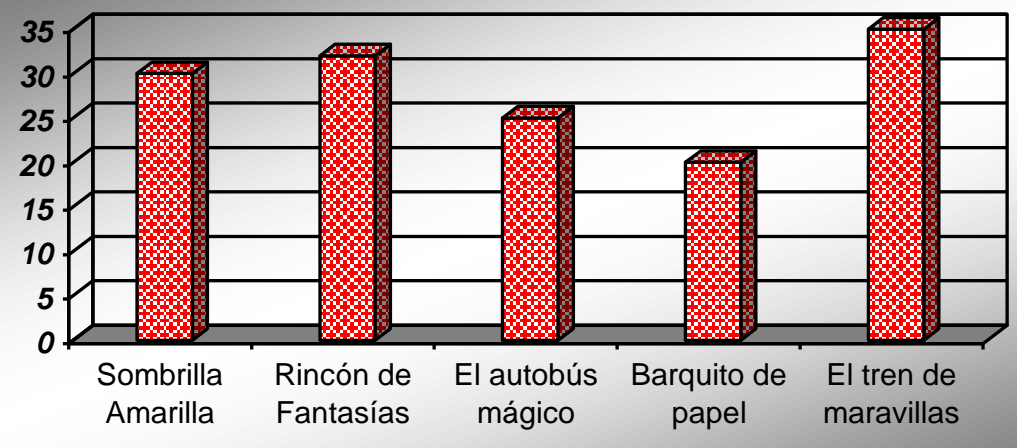

a) La mayor cantidad de alumnos prefiere:
A) __ "La sombrilla amarilla"
B) __ "El tren de maravillas"
C) __ "Barquito de papel"
D) __ "Rincón de Fantasías"

b) Los dos programas de mayor preferencia son:
A) ___ "Rincón de Fantasías" y "Barquito de papel"
B) __ "El tren de maravillas" y "La sombrilla amarilla"
C) __ "Rincón de Fantasías" y "El tren de maravillas"
D) __ "La sombrilla amarilla” y "Rincón de Fantasías"

10- En la tabla aparece representada la temperatura promedio de una semana, en la provincia Santiago de Cuba, en grados Celsius.

Semana del 18 al 24 de octubre de 2007.

\begin{tabular}{|l|c|c|c|c|c|c|c|}
\hline & \multicolumn{7}{|c|}{ Temperatura Promedio } \\
\cline { 2 - 8 } & Domingo & Lunes & Martes & Miércoles & Jueves & Viernes & Sábado \\
\hline Mínimas & 23 & 22 & 25 & 22 & 23 & 24 & 23 \\
\hline Máximas & 29 & 30 & 30 & 31 & 30 & 28 & 29 \\
\hline
\end{tabular}

1. La temperatura promedio semanal, en las tardes es:
A)
29
B) _ 207
C)
D)

2. Las temperaturas más frecuentes fueron:
A) _ $29^{\circ}$ y $22^{\circ}$
B) _ $29^{\circ}$ y $30^{\circ}$
C) _ $23^{\circ}$ y $30^{\circ}$
D) _ $22^{\circ}$ y $23^{\circ}$ 


\section{CONCLUSIÓN}

La necesidad de elevar la calidad del proceso de enseñanza aprendizaje en correspondencia con las exigencias de la sociedad, demuestra la importancia de contar con métodos de trabajo novedosos que propicien un alto nivel de desarrollo cognitivo.

El trabajo contiene un material didáctico, útil en el desempeño del profesor universitario, de los docentes en formación y de los maestros en funciones; pues les viabiliza la planificación, auto preparación y preparación de los sistemas de clases, con vista al mejor desarrollo del proceso de enseñanza-aprendizaje de que se trate.

El material didáctico ofrece recursos, a partir de un enfoque desarrollador en su concepción y se constituye en vía metodológica para todos los implicados. El mismo puede ser utilizado por la escuela en las preparaciones metodológicas, talleres, preparaciones de asignaturas, encuentros presenciales, entre otras actividades.

\section{BIBLIOGRAFÍA}

1. ACUÑA, J, Y PULIDO, K. (2016). El conocimiento didáctico-pedagógico del profesor de matemáticas, Facultad de Ciencias y Educación. Universidad distrital Francisco José de Caldas Bogotá, Colombia. Recuperado de http://repository.udistrital.edu.co/bitstream/11349/3154

2. ADDINE, F. (2004). Didáctica: teoría y práctica. La Habana. Pueblo y Educación.

3. ALBARRÄN, J. (2014). Desarrollo de capacidades matemáticas en la escuela primaria. p 39- 53. Maestría en Ciencias de la Educación: Módulo III: Primera parte: Mención en Educación Primaria. La Habana. Pueblo y Educación.

4. Algunas Exigencias para el Desarrollo y Evolución del Proceso de Enseñanza y Aprendizaje en la Escuela Primaria. (2013). ICCP. La Habana.

5. ÁRIAS, G. (2004). Folleto de Adecuaciones Curriculares para sexto grado de Matemática. Georgina Arias Leyva... [et. al]. ICCP. La Habana.

6. CASTELLANOS, D. (2002). Aprender y Enseñar en la Escuela: La Habana. Pueblo y Educación.

7. JEREZ, A. (2008). Compendio didáctico para el perfeccionamiento de la dirección del proceso de enseñanza aprendizaje en la asignatura matemática 6. grado de la escuela primaria. Tesis de Maestría. IPLAC. Santiago de Cuba.

8. JIMÉNEZ RIVERO, M. (2005). Folleto: para ti maestro. (Ejercicios de 6. grado). ICCP. La Habana.

9. LÓPEZ, E., \& MONTOYA, J. (2013). La contextualización de la didáctica de la matemática: un imperativo para la enseñanza de la Matemática en el siglo XXI. Pedagógica Universitaria, 55-56. Recuperado el 10 de Junio de 2017, de http://cvi.mes.edu.cu/peduniv/index.php/peduniv/article/view/453/447

10. LÓPEZ, P (2018). Sugerencias metodológicas para el desarrollo de la habilidad de cálculo matemático en la escuela primaria. Recuperado 16 de octubre de 2018, de Revista Opuntia Brava

11. PINTO, J, Y GONZÁLEZ, M. (2016). El conocimiento didáctico del contenido en el profesor de matemáticas: ¿una cuestión ignorada? Facultad de Educación, Universidad Autónoma de Yucatán, México. Facultad de Educación, Universidad de Salamanca, España. Recuperado de: http://www.scielo.org.mx/scielo.php?

12. RODRíGUEZ, A. (2015). Modelo del proceso de enseñanza - aprendizaje contextualizado de la asignatura Matemática. Tesis Doctoral, Granma, 82-83. Recuperado el 10 de junio de 2017. 